\title{
Estructura Factorial del Cuestionario de Estrés Percibido en la población Universitaria
}

\author{
Luciana S., Moretti* \& Leonardo Adrián, Medrano* \\ * Universidad Siglo 21
}

\begin{abstract}
Resumen.
El objetivo del presente trabajo es realizar la validación de la Escala de Estrés Percibido (CEP) de Levenstein et al. (1993) en una muestra de estudiantes universitarios cordobeses. Mediante un muestreo probabilístico intencional se seleccionaron 315 estudiantes universitarios de ambos sexos con una media de edad de 22.88 ( $\mathrm{SD}=4.26$ ). Se llevaron a cabo estudios para evaluar la confiabilidad mediante la estructura interna y consistencia interna del instrumento. A partir del análisis factorial exploratorio realizado se obtuvieron tres factores que explicaban el 50,1\% de la varianza total de la prueba: 1) Tensión y agotamiento; 2) Sobrecarga, y 3) Indefensión. Los índices de consistencia interna obtenidos a partir del Coeficiente $\alpha$ de Cronbach oscilaron entre .85 y .87, todos ellos considerados óptimos. A partir de los resultados obtenidos en las pruebas psicométricas realizadas se puede decir que el CEP es un instrumento válido y confiable para utilizarse en estudiantes universitarios. Sin embargo, estos resultados son preliminares, por lo que se requieren estudios adicionales que complementen estos hallazgos.

Palabras clave: estrés percibido; validación; confiabilidad; estudiantes universitarios.
\end{abstract}

\begin{abstract}
:
The purpose of the present study is to validate the Perceived Stress Scale (PSS, Levenstein et al., 1993) on a university student's sample. To this mean, 315 university students (male and female, $\mathrm{SD}=4.26$ ) were intentionally recruited to answer the PSS. Psychometric studies were developed to evaluate internal structure, internal consistency and external validity. Exploratory factor analysis revealed a factor structure of three dimensions that explained the 50,1\% of variance: 1) Tension and exhaustion; 2) Overburden and 3) helplessness. Additionally, Cronbach's $\alpha$ values were observed to be optimal for the three factors of the scale (among .85 and .87). The psychometric studies developed in this study suggest that the PSS is reliable and valid to be used in cordobese university students. However this study is preliminary, therefore more studies are needed to supplement these findings.
\end{abstract}

Keywords: perceived stress; validation; internal validity; university students.

\section{Introducción}

Históricamente el estrés ha sido objeto de atención e interés para la comunidad científica debido al gran impacto que esta variable ejerce sobre la salud y el funcionamiento de las personas. De manera sistemática se observa que el estrés se asocia a diversos problemas de salud tanto físicos como psicológicos: el estrés afecta el sistema inmune (Lorentz, 2006), interviene en el desarrollo de problemas somáticos tales como úlceras o inflamación en el recto (Levenstein, et al., 1993), en la disminución de la respuesta de anticuerpos (Phillips, Burns, Carroll, Ring y Drayson, 2005), entre otros factores. En una buena cantidad de investigaciones se ha encontrado que también existe evidencia que sustenta una asociación entre el estrés y los trastornos de ansiedad generalizada (Hoehn-Saric, McLeod, FunderBurk y Kowalski, 2004), con los ataques de pánico (Wood, Cano-Vindel, y Salguero, en prensa), la 
depresión (Hammen, 2005; Liu y Allow, 2010) y con problemas de naturaleza interpersonal (Levenstein, et al., 1993).

A pesar de la vasta gama de publicaciones en torno al estrés, aún persiste una gran controversia en relación a la definición conceptual, las dimensiones involucradas y los métodos para examinar dicha variable. Recientemente Karatza et al. (2014) sugirieron la existencia de tres aproximaciones básicas al estudio del estrés. En primer lugar, una aproximación ambiental circunscripta al estudio de las condiciones externas que provocan estrés (estresores). Una segunda aproximación de tipo psicológica centrada en el estudio de la percepción de estrés y su impacto sobre las emociones negativas; y, finalmente, una aproximación biológica abocada al estudio de los indicadores fisiológicos del estrés. Aunque los tres abordajes acuerdan en conceptualizar al estrés como un estado donde la homeostasis del organismo es alterada producto de una percepción real o percibida (Chrousos, 2009), persisten diferencias notables en el énfasis que se otorga a los componentes involucrados. En este sentido, algunos autores centran su atención en el estudio de los estresores externos, otros en la percepción y las respuestas de afrontamiento del organismo frente al estrés y, por último, aquellos que enfatizan el análisis y el estudio de las consecuencias que genera el estrés (Al-Sowygh, 2013). Debido a esta gran disparidad en los enfoques, es que los instrumentos desarrollados para medir esta variable también exhiben dicha discrepancia.

Según Kocalevent et al., (2007) existen más de 150 instrumentos de autoreporte que evalúan estrés, sin embargo la mayoría de ellos se focalizan en la evaluación de eventos vitales críticos, estrés cotidiano y sus concomitantes estrategias de afrontamiento y síndromes específicos asociados al estrés (como por ejemplo, burnout); dejando de lado el estudio de las percepciones subjetivas de las personas que experimentan estrés. A este respecto, el interés por la medición de las percepciones de estrés surge de la pregunta: ¿por qué un mismo estresor puede provocar experiencias diferentes de estrés en cada persona? (Karatza et al., 2014). A partir de este interrogante se comenzó a indagar sobre los factores personales, experiencias vitales, creencias e incluso factores genéticos involucrados en los procesos cognitivos que afectan la manera en que una persona percibe y responde a un 
estresor.

La importancia otorgada al componente cognitivo de la evaluación del estrés es un factor central del modelo transaccional formulado por Lazarus y Folkman (1984). De acuerdo con este modelo, el estrés puede entenderse como una relación particular entre la persona y su contexto, el cual es evaluado de modo amenazante y a partir del cual se considera desbordante respecto a los recursos con los que cuenta y que, por consiguiente, pone en riesgo su bienestar (Lazarus \& Folkman, 1984). Es preciso destacar que de acuerdo al modelo propuesto por estos autores, el estrés no es sólo un producto de un evento externo, sino que en este caso la evaluación cognitiva del individuo juega un rol central. A este respecto, la teoría transaccional del estrés entiende a la evaluación cognitiva como la atribución de significados a los acontecimientos en relación al bienestar percibido por la persona. De acuerdo con este modelo, existen tres modalidades de evaluación: la evaluación primaria, la evaluación secundaria y la reevaluación. En primera instancia, la evaluación primaria hace referencia a la evaluación cognitiva que se produce en cada transacción o relación entre el individuo y una demanda (ya sea esta interna o externa). La evaluación primaria (modo en que se define al acontecimiento activador del estrés) puede ser de cuatro tipos: percepción de amenaza, de daño o pérdida, de desafío y de beneficio; estas últimas tendrán un impacto diferencial sobre la persona dependiendo de su calidad. En segundo lugar, la evaluación secundaria implica a la valoración que hace la persona acerca de sus recursos (por ejemplo, la persona puede considerarlos deficitarios o tal vez suficientes) para hacerle frente a la situación valorada por la evaluación primaria. Es importante en este punto destacar la idea propuesta por Beck, Emery y Greenberg (2014) acerca de que ambas, la evaluación primaria y la secundaria, se producen en simultáneo y de manera casi automática. En última instancia, la reevaluación involucra una retroalimentación de información acerca de los eventos y afrontamientos previos, lo cual permite realizar ajustes respecto a valoraciones anteriores (González-Ramirez \& Landero Hernández, 2008).

Si bien la percepción de estrés es un elemento central para el modelo transaccional, sólo se han desarrollado pocos instrumentos estandarizados para evaluar la manera en que las personas perciben situaciones de estrés y sus consecuencias (Kocaleventa et al., 2007). Algunos de los instrumentos desarrollados son: el Stress Appraisal Measure (Peackock y Wong, 1990), el Impact of Event Scale (Horowitz, Wilner y Alvarez, 1979) y la Escala Global de Estrés Percibido (Cohen, Kamarck y Mermelstein, 1983). De entre los 
instrumentos desarrollados, uno de los que cuenta con mayor apoyo empírico es el Cuestionario de Estrés Percibido (CEP) de Levenstein et al. (1993). Este instrumento construido por Levestein et al. (1993) para ser utilizado inicialmente en el contexto para desarrollar estudios en el ámbito de la investigación psicosomática. Sin embargo su utilización se ha extendido en diversos ámbitos de ejercicio profesional, tanto en población clínica como no clínica (Kocalevent et al., 2007; Krohn, et al., 2011; Wikgren et al., 2012).

El CEP se desarrolló originalmente con el fin de brindar una visión alternativa al estudio del estrés y su impacto a nivel somático. Según Levenstein et al. (1993) muchos trabajos se limitaban a correlacionar la existencia de eventos vitales estresantes y su relación con los síntomas somáticos, sin considerar las impresiones y percepciones de los pacientes. Fue a partir de ello que Levenstein et al. (1993) desarrollaron un instrumento con el fin de evaluar el estrés percibido en una situación específica, teniendo en cuenta las respuestas cognitivas y emocionales que experimenta la persona frente a una situación estresante. Para ello, y a través de entrevistas cualitativas a pacientes con problemas somáticos (úlceras y asma principalmente), se redactaron 60 ítems. Luego de una prueba piloto se administró una versión de 36 ítems a una muestra de 48 pacientes a partir de la cual se eliminaron 6 reactivos. La versión final de 30 ítems fue analizada en una muestra bilingüe (inglés e italiano) de 230 pacientes con un análisis factorial exploratorio. A partir de este análisis los reactivos se agruparon en 7 factores subyacentes que explicaban un $60 \%$ de la varianza: acoso (15\%), sobrecarga (13\%), irritabilidad (8\%), falta de alegría $(6 \%)$, preocupación (8\%), fatiga (2\%), y tensión (8\%) (Levenstein et al., 1993).

El CEP constituye uno de los instrumentos más utilizados a nivel mundial para evaluar estrés percibido. A este respecto, se pueden encontrar validaciones de esta escala en población Alemana (Fliege, Rose, Arck, Levenstein y Klapp, 2001), Italiana (Levenstein et al., 1993), Española (Sanz-Carillo et al., 2002) y Griega (Karatza et al., 2014). Los resultados obtenidos en dichas validaciones no han sido homogéneos, sobre todo en lo respecta a la estructura factorial del instrumento. La versión española, por ejemplo, obtiene 7 factores subyacentes que explican un 58\% de la varianza: 1) tensión-fatiga, 2) conflicto-aceptación social, 3) energía-disfrute, 4) sobrecarga-acoso, 
5) autorrealización-satisfacción, 6) miedo-ansiedad y 7) factor sin denominación (SanzCarillo et al., 2002). Como puede apreciarse, los factores obtenidos en la versión española no se corresponden con la versión original y, además, el último factor sólo incluye un reactivo de alta saturación, por lo cual el factor 7 fue descartado. Es preciso señalar que en la validación desarrollada por Sanz-Carrillo et al. (2002) se trabajó con una muestra de 174 casos, tamaño muestral inferior al sugerido por la literatura psicométrica (Hogan, 2004).

Por otra parte, la versión griega (Karatza et al., 2014) también ha mostrado resultados discordantes con la versión original. En este estudio $(\mathrm{N}=1318)$ se llevaron a cabo estudios de análisis factorial exploratorio y confirmatorio. En primer lugar, en la fase exploratoria, los autores identificaron cinco factores subyacentes que explicaban un 54\% de la varianza total: 1) sobrecarga, 2) preocupación, 3) disfrute, 4) tensión-fatiga y 5) acoso. Posteriormente, mediante una aproximación confirmatoria comparan el ajuste del modelo de 5 factores y el modelo de 7 factores. Ninguno de los dos modelos presentó un ajuste óptimo a los datos, aunque el modelo de 5 factores presentó un ajuste superior $(\mathrm{CFI}=.85 ; \mathrm{AGFI}=.83 ; \mathrm{NFI}=.83$; RMSEA=.06).

En última instancia, la versión alemana del CEP (Fliege et al., 2001; N=650) identifica sólo cuatro factores subyacentes: 1) preocupación, 2) tensión, 3) disfrute y 4) demandas. Cabe destacar que los autores descartaron 10 reactivos por presentar baja saturación factorial, por lo que la escala quedó finalmente conformada por 20 ítems.

Un aspecto discutible tanto de los estudios de validación (Karatza et al., 2014; SanzCarillo et al., 2002) como del estudio original (Levenstein et al., 1993) es el criterio seleccionado para determinar la cantidad de factores subyacentes. A este respecto, las versiones citadas emplean como único criterio de extracción de factores la regla Kaiser de autovalores superiores a 1 . Tal como señala la literatura, el empleo de un único criterio puede conducir a distorsionar el número real de factores, más aún si se utiliza como único criterio la regla Kaiser dado que tiende a sobreestimar la cantidad de factores subyacentes (Pérez y Medrano, 2011). Sólo recientemente se han realizado estudios que contemplen criterios adicionales para determinar la cantidad de factores subyacentes en el CEP. De esta manera, Montero-Marin, Demarzo, Pereira, Olea y García-Campayo (2014) trabajaron con una muestra de 314 estudiantes de odontología con la cual efectuaron análisis del CEP considerando como criterio adicional el análisis factorial paralelo. Los autores concluyeron, mediante un análisis factorial confirmatorio, que el CEP poseía una estructura jerárquica 
donde había un factor de segundo orden denominado "estrés percibido" y dos factores de primer orden: 1) frustración (el cual involucra elementos originalmente incluidos en las escalas de falta de alegría y preocupaciones), y 2) tensión (incluye principalmente ítems de las escalas de tensión y sobrecarga).

El presente trabajo se desarrolló con una doble finalidad, en primer lugar efectuar análisis complementarios que permitan clarificar la estructura factorial subyacente al CEP y, en segundo lugar, evaluar sus propiedades psicométricas en la población universitaria de Argentina atendiendo a la ausencia de estudios psicométricos de esta escala en la población local.

\section{Método}

\section{Participantes}

Se administró el CEP a una muestra no probabilística accidental compuesta por 315 estudiantes universitarios de diferentes carreras con edades comprendidas entre los $16 \mathrm{y}$ 25 años (media $=22.88$; desviación estándar $=4.26$ ) de ambos géneros (hombres $=32.4 \%$; mujeres $=67.3 \%)$. Cabe señalar que los estudiantes accedieron voluntariamente a participar y luego de haber brindado su consentimiento informado.

\section{Instrumentos}

Escala de Estrés Percibido (CEP): instrumento desarrollado por Levenstein et al. (1993) con el fin de evaluar el nivel auto-percibido de estrés. Para ello los participantes deben responder con una escala de tipo Likert de 4 puntos ( $1=$ "casi nunca" a 4= "usualmente") la frecuencia con que experimentan respuestas cognitivas y emocionales asociadas al estrés. La escala original incluye 30 reactivos, no obstante estudios posteriores de validación modificaron la cantidad de ítems del CEP (Fliege et al., 2001; Sanz-Carillo et al., 2002), al igual que aún no se ha determinado con exactitud la cantidad de factores subyacentes (variando el número de factores subyacentes de 7 en el estudio de Levenstein et al., 1993; y a 2 factores en el trabajo de Montero-Marin et al., 2014). Más allá de la discrepancia en cuanto a las 
dimensiones subyacentes del CEP, este instrumento ha mostrado adecuadas propiedades psicométricas en términos de consistencia interna, validez convergente y validez predictiva (Montero-Marin et al., 2014; Karatza et al., 2014; Sanz-Carillo et al., 2002). En el presente estudio se trabajó con la versión española del CEP (Sanz-Carillo et al., 2002).

\section{Procedimiento}

Antes de administrar el CEP a la muestra definitiva se realizó una prueba piloto y se efectuaron ajustes en algunas expresiones idiomáticas, dado que la versión Española (SanzCarillo et al., 2002), utilizaba un vocabulario diferente al utilizado por los universitarios argentinos. Posteriormente, se recabaron los datos en la muestra definitiva y se realizaron estudios de análisis factorial exploratorio utilizando el programa SPSS versión 20. Adicionalmente, para el cálculo del análisis factorial paralelo se utilizó el software desarrollado por Watkins (2008). En última instancia, se analizó la consistencia interna de cada sub-escala mediante el coeficiente alfa de Cronbach.

\section{Resultados.}

Al realizar el análisis de exploración inicial no se observaron variables con más del 5\% de datos ausentes, ni la existencia de casos atípicos univariados (valores fuera del rango z \pm 3 ). Todos los ítems presentaron una distribución cercana a la normalidad con valores de asimetría y curtosis inferiores a \pm 2 . Finalmente, se comprobó la ausencia de multicolinealidad al no encontrar correlaciones inter-ítem superiores o iguales a .90 (Tabachnick y Fidell, 2001).

Para determinar la factibilidad del análisis factorial exploratorio se calculó el índice de adecuación muestral Kaiser-Meyer-Olkin $(\mathrm{KMO}=.92)$, y la prueba de esfericidad de Bartlett $\left(\chi^{2}=3820,46 ; \mathrm{gl}=435 ; p<.000\right)$, observándose una intercorrelación óptima para efectuar el análisis factorial. Para realizar el análisis factorial exploratorio se usó el método de estimación de Máxima Verosimilitud y con el fin de determinar la cantidad de factores subyacentes se consideraron múltiples criterios: 1) proporción de varianza explicada, 2) gráfico de sedimentación, 3) análisis factorial paralelo y, finalmente, 4) coherencia teórica en la agrupación de reactivos. La regla Kaiser de autovalores superiores a 1 sugería la extracción de 6 factores que explicaban un 60,15\% de la varianza total. No obstante, al examinar el gráfico de sedimentación se identifican 3 factores subyacentes (figura 1). Se observaron 
resultados semejantes al realizar el análisis factorial paralelo. A partir de variables no correlacionadas generadas por simulación Montecarlo (se simularon 500 muestras) se observó que el cuarto factor presentaba una media de autovalor superior al autovalor del cuarto factor de la solución observada (media de autovalor en datos simulados $=1,42$; autovalor en datos observados $=1,32$ ). De esta manera se obtiene evidencia que sugiere que la contribución del cuarto factor no es superior a la contribución que se obtendría por azar, sugiriendo así la extracción de 3 factores.

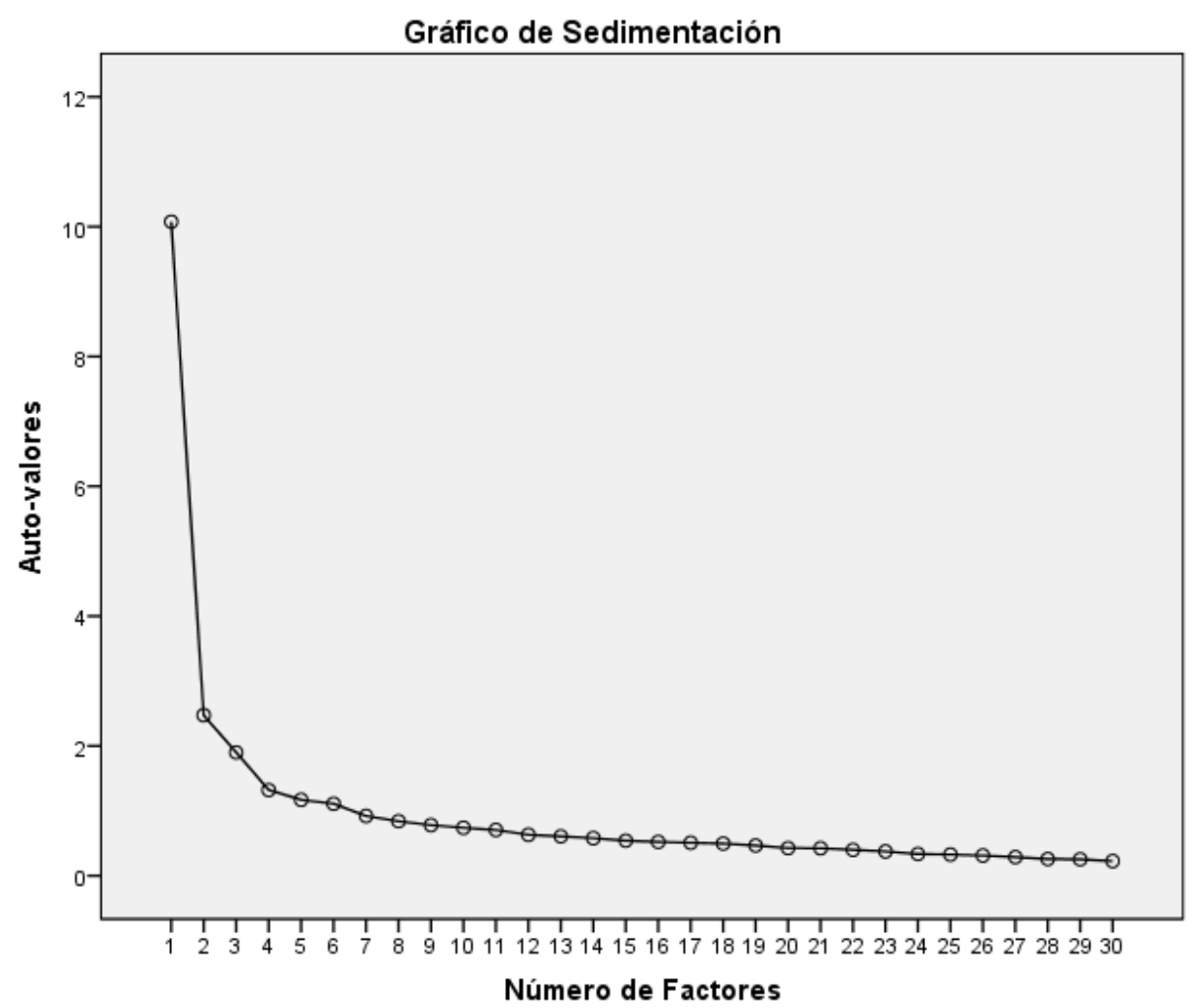

Figura 1. Gráfico de Sedimentación del CEP.

A partir de lo mencionado, se extrajeron tres factores y se realizó una rotación Promax para interpretarlos debido a que éstos presentaban correlaciones superiores a ,30 (valores $r$ comprendidos entre 49 y ,58). Por otro lado, se procedió a eliminar 2 reactivos, el número 3 ("está irritable y mal humorado") y el 17 ("Se siente seguro y protegido") dado que presentaban saturaciones inferiores a .30 en los tres factores. En la tabla 1 se presenta la matriz rotada, donde puede apreciarse la saturación factorial de los reactivos del CEP en tres 
factores que explican en conjunto un 50,1\% de la varianza total de la prueba. Si bien algunos reactivos presentaron un comportamiento multidimensional, se optó por retenerlos a partir de criterios teóricos.

Tabla 1. Estructura factorial del CEP (matriz rotada)

\begin{tabular}{|c|c|c|c|}
\hline Ítems del CEP & $\begin{array}{c}\text { Tensión y } \\
\text { Agotamiento } \\
\quad(\alpha=, 87)\end{array}$ & $\begin{array}{l}\text { Sobrecarga } \\
\qquad(\alpha=, 76)\end{array}$ & $\begin{array}{l}\text { Indefensión } \\
\qquad(\alpha=, 85)\end{array}$ \\
\hline Se siente descansado & -.609 & & \\
\hline Se divierte & -.805 & & \\
\hline Se siente alegre & -.746 & & \\
\hline Tiene problemas para relajarse & .642 & & \\
\hline Se siente tranquilo & .664 & & \\
\hline Tiene tiempo suficiente para usted & -.496 & -.457 & \\
\hline Se siente lleno de energía & -.807 & & \\
\hline Se siente tenso & .475 & & \\
\hline Se siente cansado & .493 & .359 & \\
\hline Siente que está haciendo cosas que realmente le gustan & -.523 & & -.376 \\
\hline Tiene demasiadas cosas que hacer & & .787 & \\
\hline Se siente agobiado por la responsabilidad & & .620 & \\
\hline Se siente presionado por los plazos de tiempo & & .610 & \\
\hline Tiene que tomar demasiadas decisiones & -.317 & .788 & \\
\hline Siente que se le hacen demasiadas peticiones & & .424 & \\
\hline Se siente agotado mentalmente & .316 & .412 & \\
\hline Siente que tiene prisa & & .626 & \\
\hline Tiene muchas preocupaciones & & .628 & \\
\hline Se siente solo o aislado & & & .702 \\
\hline Tiene miedo del futuro & & & .572 \\
\hline Siente que hace cosas por obligación, no porque quiera & & & .575 \\
\hline \multicolumn{4}{|l|}{ hacerlas } \\
\hline Se siente criticado o juzgado & & & .715 \\
\hline Esta bajo la presión de otras personas & & .450 & 619 \\
\hline Se siente desanimado & .428 & & 477 \\
\hline Sus problemas parecen multiplicarse & & .371 & .419 \\
\hline Teme que no pueda alcanzar todas sus metas & & & .551 \\
\hline Se siente frustrado & & & .759 \\
\hline Se encuentra sometido a situaciones conflictivas & & .335 & .571 \\
\hline
\end{tabular}


A partir de la distribución de los reactivos se efectuó una interpretación de los factores subyacentes que fueron identificados como: 1) Tensión y agotamiento $(\alpha=, 87)$, esta dimensión involucra 10 reactivos que denotan niveles de agotamiento, falta de energía y percepción de estados de tensión; 2) Sobrecarga $(\alpha=, 87)$, este factor está compuesto por 8 ítems que expresan percepción de sobrecarga en tareas, percepción de falta de tiempo y de demandas externas, y 3$)$ Indefensión $(\alpha=, 85)$, esta dimensión describe circunstancias de la persona que son evaluadas como estados de indefensión, desesperanza y frustración.

\section{Discusión.}

La percepción de estrés ocurre cuando la persona percibe que una demanda del entorno excede los recursos personales de afrontamiento y amenazan a su propio bienestar (Montero-Marin et al., 2014). De acuerdo con lo que señalan Polanco-Roman y Miranda (2013), la etapa por la que atraviesan los jóvenes universitarios, comprendida entre los 18 y 25 años de edad, resulta especialmente crítica debido a que durante este período de emergencia de la adultez se hacen frente a nuevas demandas y estresores, aumentando la vulnerabilidad a experimentar problemas mentales asociados al estrés. En este sentido, la transición a los estudios superiores es un evento estresante ya que los estudiantes se enfrentan con múltiples estresores como la sobrecarga académica, la presión constante de progresar académicamente, la competencia con los pares y, en el contexto local especialmente, la carga financiera que implica llevar adelante una carrera universitaria (Lee, Dickson, Conley y Holmbeck, 2014). Ante este panorama, muchos estudiantes se ajustan de un modo efectivo al contexto universitario mientras que otros sufren un impacto adverso por el estrés que genera transitar por esta etapa y, consecuentemente, muchos de ellos pueden desarrollar desórdenes psicológicos (Bewick, Koutsopoulou, Miles, Slaa \& Barkham, 2010). Es por ello que resulta de importancia contar con instrumentos adaptados a la población local de universitarios que permitan establecer diagnósticos precisos de poblaciones universitarias posiblemente vulnerables.

El CEP constituye uno de los instrumentos de mayor uso para evaluar la percepción de estrés. Aunque este instrumento ha demostrado una adecuada capacidad predictiva (Montero-Marin et al., 2014), no existe consenso en relación a la estructura factorial 
subyacente. Si bien los autores originales del CEP identificaron 7 factores subyacentes (Levenstein et al., 1993), este número ha variado sustancialmente en las posteriores validaciones. Así, la versión española de Sanz-Carillo et al. (2002) cuenta con 6 factores, la versión griega (Karatza et al., 2014) incluye 5 factores y la versión alemana (Fliege et al., 2001) considera 4 factores. Como se comentó con anterioridad dichos estudios sólo utilizaron como criterio para determinar la cantidad de factores la regla Kaiser, la cual tiende a sobreestimar el número de factores.

En relación a este último punto, el trabajo de Montero-Marin et al. (2014) constituye una excepción dado que los autores consideraron múltiples criterios en la extracción de factores y concluyeron que el CEP comprende un factor de estrés general de segundo orden y dos factores específicos de primer orden: tensión y frustración. Aunque este último estudio es más completo en términos metodológicos, posee algunas limitaciones teóricas. De hecho, los autores no justifican teóricamente el agrupamiento de los reactivos en dos dimensiones y, además, incluyen reactivos no descriptivos de dichas dimensiones. Los autores denominan "frustración" a uno de los factores cuando en realidad algunos reactivos no reflejan sentimientos o estados de frustración: "sentirse solo" o "sentirse desprotegido", por ejemplo. Quizás una denominación más pertinente para este factor sería la de "indefensión" ya que no sólo se reflejan sentimientos de frustración, sino también de desesperanza y falta de motivación. Por otra parte, según Montero-Marin et al. (2014) la dimensión “tensión” de la escala incluye ítems de sobrecarga y tensión, sin embargo puede observarse que también aparecen reactivos que involucran estados de fatiga y agotamiento ("me siento cansado", "me siento mentalmente exhausto", por ejemplo).

El presente trabajo se observó que los reactivos del CEP podrían agruparse en tres dimensiones íntegramente asociadas con el estrés. Estas dimensiones fueron interpretadas como: 1) tensión y agotamiento, 2) sobrecarga e 3) indefensión. Al examinar dichas dimensiones se hipotetiza que estos factores podrían interpretarse considerando el proceso implicado en el estrés. En efecto debe considerarse que los ítems provienen de afirmaciones que diferentes pacientes realizan acerca de percepciones subjetivas que poseen sobre sus reaccinoes de estrés. En este sentido es altamente probable que éstos pacientes se encuentren en diferentes "fases" del estrés. A partir de los resultados obtenidos se hipotetiza que los factores obtenidos podrían reflejar dichas fases. De esta forma podría sugerirse que en primera instancia las personas perciben sobrecarga, es decir que las demandas exceden los 
recursos que poseen para poder afrontarlas. A continuación existiría una fase de tensión y agotamiento, durante la cual las personas deben esforzarse por encima de sus recursos para poder responder a estas demandas (tensión) lo cual trae aparejado sentimientos de cansancio que ocurren como consecuencia de esta sobre-exigencias (agotamiento). El proceso puede finalizar en esta etapa si la persona logra responder eficazmente a la demanda, sin embargo en caso de no lograrlo ocurre una tercera fase caracterizada por la percepción de incontrabilidad sobre los estímulos, sentimientos de desesperanza, frustración y desánimo (figura 2).

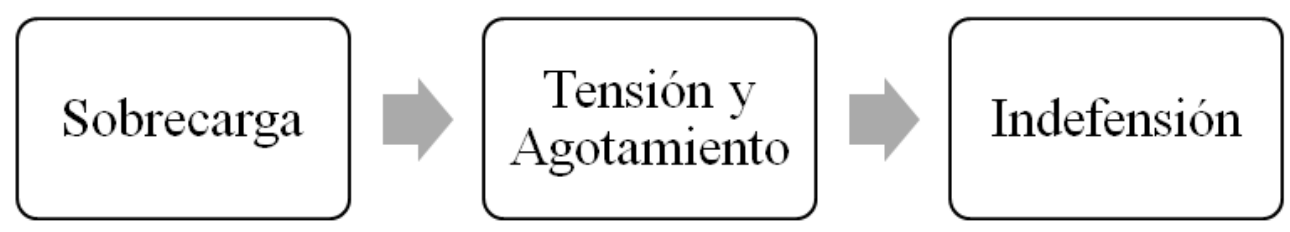

Figura 2. Proceso hipotetizado del estrés percibido.

Es importante señalar que desde esta propuesta no todas las personas necesariamente deben atraversar por todas las fases de estrés. Algunos simplemente podrían reportar sentimientos de sobrecarga, otros de tensión, y sólo en aquellos casos donde exista mayor cronicidad se observarían los síntomas de indefensión.

Si bien el presente modelo es sólo un modelo tentativo, resulta un modelo plausible más aún tomando en consideración la literatura previa sobre el tema. Tal como señala Overmeir (2009), las respuestas de indefensión aparecen cuando los sujetos perciben que no poseen control sobre los estresores. En los experimentos iniciales se exponía a una serie de eventos aversivos impredecibles e incontrolables, tras fallas repetidas en los intentos de controlar los estresores aparecían respuestas cognitivas y emocionales negativas, destacándose principalmente la dificultad para aprender nuevos comportamientos. Aunque Seligman (1975) ha ligado los estados de indefensión principalmente a sintomatología depresiva, otros autores señalan que la indefensión aprendida es esencialmente un fenómeno de estrés. De esta manera la indefensión es una consecuencia de una exposición extendida a estímulos aversivos incontrolables.

Frente a fallas sistemáticas en los intentos por afrontar los estresores aparecen los 
estados de indefensión ya que las personas no perciben contingencias entre su propio comportamiento y la respuesta a demandas o exigencias a las que se ven expuestos. La desesperanza es una consecuencia típica de los estados de indefensión, ya que se desarrolla la creencia de que la falta de control continuará en el futuro, más aún desarrollan la tendencia a esperar relaciones no contingentes relaciones que de contingencia (Barraza Macías y Silerio Quiñonez, 2012). La expectativa de incontrolabilidad y la desesperanza interfieren con la adquisición de nuevos aprendizajes, y generan déficit motivacionales y emocionales, entre otros (Docampo, 2002). Cabe destacar que la desesperanza es un factor de riesgo asociado al comportamiento suicida. En efecto, en un estudio reciente desarrollado por Polanco-Roman y Miranda (2013) se observó que la desesperanza constituía un factor que moderaba la relación entre estrés, depresión e ideación suicida.

Por otra parte, este modelo podría explicar la relación variable existente entre estrés, ansiedad y depresión. En efecto podría hipotetizarse que durante las dos primeras fases de estrés se observa una mayor prevalencia de ansiedad, mientras que en la tercera fase una mayor prevalencia de depresión. A este respecto, Stewart-Brown et al. (2000) afirman que exhibir niveles elevados de estrés aumenta el riesgo que una persona desarrolle ansiedad y depresión. Aunque aún no se encuentra claramente delimitada una explicación sobre la asociación entre estos factores, son abundantes los estudios que corroboran dichas correlciones (Eisenbarth, Champeau, \& Donatelle, 2013; Hammen, 2005; Liu y Allow, 2010).

A pesar de que el modelo planteado es plausible y coherente en términos teóricos se requieren de investigaciones más controladas para verificar si el modelo tiene el comportamiento causal hipotetizado. Sin embargo resulta un modelo promisorio y de mayor parsimonia que los modelos anteriormente propuestos para el CEP (Levenstein et al., 1993; Karatza et al., 2014; Montero-Marin et al., 2014; Sanz-Carillo et al., 2002).

En relación a las implicancias prácticas resta por señalar que el CEP es un instrumento inicialmente válido y confiable para ser utilizado en la población universitaria. Este es un aspecto importante ya que los estudiantes universitarios son un grupo de alto riesgo, mostrando una prevalencia alta de trastornos psicológicos en relación a la población general. Un estudio desarrollado por Stallman (2010) encontró que los estudiantes universitarios eran uno de los grupos de la población Australiana que mostraban mayor riesgo de desarrollar desórdenes psicológicos. Este estudio reveló que el 83.9\% de los estudiantes 
universitarios reportaron niveles elevados de malestar psicológico, incluyendo estrés, depresión y estados ansiosos. A partir del presente estudio se cuenta con un inventario validado para evaluar el estrés percibido en la población universitaria local, lo cual permitiría a futuro identificar estudiantes en riesgo, examinar variables asociadas al estrés y evaluar la eficacia de intervenciones psicológicas destinadas a disminuir el estrés percibido en esta población.

\section{Referencias}

Al-Sowygh, Z. H. (2013). Academic distress, perceived stress and coping strategies among dental students in Saudi Arabia. The Saudi Dental Journal, 25, 97-105. http://dx.doi.org/10.1016/j.sdentj.2013.05.002

Barraza Macías, A. \& Silerio Quiñonez, J. (2012).Indefensión escolar aprendida en alumnos de educación media superior y su relación con dos indicadores del desempeño académico. Psicogente, 15 (28), 337-347.

Beck, A. T.; Emery, G. \& Greenberg, R. (2014). Trastornos de ansiedad y fobias. Una perspectiva cognitiva.

Bewick, B.; Koutsopoulou, G.; Miles, J.; Slaa, E. \& Barkham, M. (2010) Changes in Undergraduate Students' Psychological Wellbeing as They Progress through University. Studies in Higher Education, 35, 633-645.

Cohen, S., Kamarck, T., \& Mermelstein, R. (1983). A global measure of perceived stress. Journal of Health and Social Behavior, 24, 385-396. http://dx.doi.org/10.2307/2136404

Chrousos, G. P. (2009). Stress and disorders of the stress system. Nature Reviews Endocrinology, 5, 374-381. doi:10.1038/nrendo.2009.106

Docampo, M. (2002). Diferencias de género en las explicaciones causales de adolescentes. Psicothema, 14, 572-576.

Eisenbarth, C. A., Champeau, D. A. \& Donatelle, R. J. (2013). Relationship of Appraised Stress, Coping Strategies, and Negative Affect among College Students. International Journal of Psychology and Behavioral Sciences, 3, 131-138.

Fliege, H., Rose, M., Arck, P., Levenstein, S., \& Klapp, B. F. (2001). Validierung des "Perceived Stress Questionnaire”, (PSQ) an einerdeutschen strichprobe. Diagnostica, 
47, 142-152. http://dx.doi.org/10.1026//0012-1924.47.3.142

González Ramírez, M. T. \& Landero Hernández, R. (2008). Confirmación de un modelo explicativo del estrés y de los síntomas psicosomáticos mediante ecuaciones estructurales. Rev Panam Salud Publica, 23 (1), 7-18.

Hammen, C. (2005). Stress and depression. Annual Review of Clinical Psychology, 1, $293-$ 319. doi: 10.1146/annurev.clinpsy.1.102803.143938

Hammen, C.; Brennan, P. A. \& Shih, J. H. (2004) Family Discord and Stress Predictors of Depression and Other Disorders in Adolescent Children of Depressed and Nondepressed Women. Journal of the American Academy of Child \& Adolescent Psychiatry, 43, 994-1002.

Hoehn-Saric, R., McLeod, D. R., FunderBurk, F. \& Kowalski, P. (2004). Somatic symptoms and physiologic responses in generalized anxiety disorderand panic disorder. An ambulatory monitor study. Arch Gen Psychiatry. 61 (9), 913-921. doi:10.1001/archpsyc.61.9.913.

Hogan, T. (2004). Pruebas Psicológicas. México: Manual Moderno.

Horowitz, M. J., Wilner, N., \& Alvarez, W. (1979). Impact of event scale: A measure of subjective distress. Psychosomatic Medicine, 41, 209-218. http://dx.doi.org/10.1097/00006842-197905000-00004

Karatza, E., Kourou, D., Galanakis, M., Varvogli, L., \& Darviri, C. (2014). Validation of the greek version of perceived stress questionnaire: Psychometric properties and factor structure in a population-based survey. Psychology, 5, 1268-1284. http://dx.doi.org/10.4236/psych.2014.510139

Kocalevent, R. D., Levenstein, S., Fliege, H., Schmid, G., Hinz, A., Brähler, E., \&Klapp, B. F. (2007). Contribution to the construct validity of the Perceived Stress Questionnaire from a population-based survey. Journal of psychosomatic research, 63(1), 71-81.

Krohn, M., Listing, M., Tjahjono, G., Reisshauer, A., Peters, E., Klapp, F. B., \& Rauchfuss, M. (2011). Depression, mood, stress, and Th1/ Th2 immune balance in primary breast cancer patients undergoing classical massage therapy. Supportive Care in Cancer, 19, 1303-1311. http://dx.doi.org/10.1007/s00520-010-0946-2

Lazarus, R. S. \& Folkman, S. (1984). Estrés y Procesos Cognitivos. Barcelona: Martínez Roca.

Lee, C.; Dickson, D. A.; Conley, C. S. \& Holmbeck, G. N. (2014) A Closer Look at Self- 
Esteem, Perceived Social Support, and Coping Strategy: A Prospective Study of Depressive Symptomatology across the Transition to College. Journal of Social and Clinical Psychology, 33, 560-585.

Levenstein, S., Prantera, C., Varvo, V., Scribano, M. L., Berto, E., Luzi, C., \&Andreoli, A. (1993). Development of the Perceived Stress Questionnaire: a new tool for psychosomatic research. Journal of Psychosomaticresearch, 37 (1), 19-32.

Linton, R. (2014). Personal and Work Stressors as Possible Predictors of Feelings of Hopelessness in Law Enforcement(Doctoral dissertation, Alliant International University).

Liu, R. T., \& Alloy, L. B. (2010). Stress generationin depression: A systematic review of theempirical literature and recommendationsfor future study. Clinical Psychology Review, 30, 582-593.

Lorentz, M. (2006). Stress and psychoneuroimmunology revised: Using mind-body intervention to reduce stress. Alternative Journal of Nursing, (11), 1-11.

Montero-Marin, J., Piva Demarzo, M. M., Pereira, J. P., Olea, M., \& García-Campayo, J. (2014). Reassessment of the psychometric characteristics and factor structure of the 'Perceived Stress Questionnaire' (PSQ): Analysis in a sample of dental students. PLoS ONE, 9 (1). doi:10.1371/journal.pone.0087071

Peackock, E. J. \& Wong, P. T. P. (1990). The stress appraisal measure (SAM): A multidimensional approach to cognitive appraisal. Stress Medicine, 6, 227-236.

Perez, E. R. \& Medrano, L. (2011). Análisis factorial exploratorio: Bases conceptuales y metodológicas. Revista Argentina de Ciencias del Comportamiento, 2 (1), 58-66.

Phillips, A. C., Burns, V. E., Carroll, D., Ring, C., \& Drayson, M. (2005). The association between life events, social support, and antibody status following thymus-dependent and thymus-independent vaccinations in healthy young adults. Brain, behavior, and immunity, 19(4), 325-333.

Polanco-Roman, L., \& Miranda, R. (2013). Culturally-related stress, hopelessness, and vulnerability to depressive symptoms and suicidal ideation in emerging adulthood. Behavior Therapy, 44, 75-87.

Sanz-Carrillo, C., Garcia-Campayo, J., Rubio, A., Santed, M. A., \& Montoro, M. (2002). Validation of the spanish version of the Perceived Stress Questionnaire. Journal of 
Psychosomatic Research, 52, 167-172. http://dx.doi.org/10.1016/S00223999(01)00275-6

Seligman, M. E. P. (1975). Helplessness: On depression, development, and death. San Francisco: Freeman.

Stallman, H. M. (2010) Psychological Distress in University Students: A Comparison with General Population Data. Australian Psychologist, 45, 249-257.

Stewart-Brown, S.; Evans, J.; Patterson, J.; Petersen, S.; Doll, H.; Balding, J. \& Regis, D. (2000) The Health of Students in Institutes of Higher Education: An Important and Neglected Public Health Problem. Journal of Public Health Medicine, 22, 492.

Tabachnick, B. \& Fidell, L. (2001). Using multivariate statistics. New York: Harper \& Row.

Watkins, M. (2008). Monte Carlo for PCA parallel analysis (Version 2.3) [Computer software].

Wikgren, M., Maripuu, M., Karlsson, T., Nordfall, K., Bergdahl, J., Hultdin, J., Del-Favero, J., Roos, G., Nilsson, L. G., Adolfsson, R., \& Norrback, K. F. (2012). Short telomeres in depression and the general population are associated with a hypocortisolemic state. Biological Psychiatry, 71, 294-300. http://dx.doi.org/10.1016/j.biopsych.2011.09.015

Wood, C. M., Cano-Vindel, A., \& Salguero, J. M. (en prensa). A diathesis-stress model of panic disorder in the general population: the mediator role of physiological anxiety and physical concerns. Anxiety, Stress \& Coping. 